\title{
Addendum: Omission of the Description of Informed Consent on the Identifiable Photos and the Description of Institutional Review Board Number
}

Editorial Office, Journal of Neurosonology and Neuroimaging

1021 Tongil-ro, Eunpyeong-gu, Seoul, 03312, Rep. of Korea

Tel: +82-2-2030-4616, E-mail: ksn@neurosonology.or.kr, Website: http://www.j-nn.org

Out of 26 reviews, original articles, or case reports published in Journal of Neurosonology and Neuroimaging from two issues in 2019, 1 review article lacked the description of informed consent, in which identifiable photos were present. Also, 1 original article lacked the description of institutional review board number. Regarding the former article, however the editorial office confirmed that the corresponding author of the review article had already received the informed consent before submitting the manuscript. Therefore, editorial office apologizes readers not checking those ethical issues and publishes Addendum as follows:

1. Pictorial Essay: Transcranial Doppler Findings of the Intracranial and Extracranial Diseases Jongyeol Kim

J Neurosonol Neuroimag 2019;11(1):1-21. https://doi.org/10.31728/jnn.2018.00039

Note

PATIENT CONSENT

The patient provided written informed consent for the publication and the use of their images.

2. Decreased Bone Marrow Activity Measured by Using 18F-fluorodeoxyglucose Positron Emission Tomography among Patients with Cerebral Atherosclerosis

Jeong-Min Kim, Eun Seong Lee, Kwang-Yeol Park, Ju Won Seok, Oh-Sang Kwon

2019;11(1):78-83. https://doi.org/10.31728/jnn.2018.00028

Note

INSTITUTIONAL REVIEW BOARD (IRB) NUMBER

Since the study was reviewed and approved by the IRB of an an university hospital, IRB number was added for the clarity. 